\title{
IdeAs
}

Idées d'Amériques

13 | 2019

La photographie documentaire contemporaine dans les Amériques

\section{Guillaume Marche, La militance LGBT aux États-Unis : sexualité et subjectivité}

Lyon, Presses universitaires de Lyon, 2017, 304 pages

\section{Julie Podmore}

\section{OpenEdition \\ Journals}

\section{Édition électronique}

URL : http://journals.openedition.org/ideas/5423

DOI : $10.4000 /$ ideas.5423

ISSN : 1950-5701

\section{Éditeur}

Institut des Amériques

\section{Référence électronique}

Julie Podmore, "Guillaume Marche, La militance LGBT aux États-Unis : sexualité et subjectivité », IdeAs

[En ligne], 13 | 2019, mis en ligne le 01 mars 2019, consulté le 24 septembre 2020. URL : http:// journals.openedition.org/ideas/5423 ; DOI : https://doi.org/10.4000/ideas.5423

Ce document a été généré automatiquement le 24 septembre 2020.

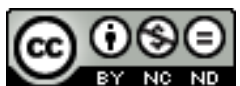

IdeAs - Idées d'Amériques est mis à disposition selon les termes de la licence Creative Commons Attribution - Pas d'Utilisation Commerciale - Pas de Modification 4.0 International. 


\section{Guillaume Marche, La militance LGBT aux États-Unis : sexualité et subjectivité}

Lyon, Presses universitaires de Lyon, 2017, 304 pages

Julie Podmore

\section{RÉFÉRENCE}

Guillaume Marche, La militance LGBT aux États-Unis : sexualité et subjectivité, Lyon, Presses universitaires de Lyon, 2017, 304 pages

1 Le mouvement LGBT américain a-t-il perdu plus qu'il n'a gagné ? En dépit de toutes ses réussites institutionnelles, commerciales et législatives au cours des quarante dernières années, le mouvement a-t-il manqué l'occasion d'instaurer la libération sexuelle? Malgré toutes les promesses radicales de la politique queer au début des années 1990, comment et à quel moment le pouvoir transformateur de ce mouvement social est-il perdu ? Ce sont les questions que Guillaume Marche aborde dans La militance LGBT aux États-Unis. En réfléchissant aux cinquante années entre 1960 et 2010, il conclut que les résultats de l'activisme LGBT américain sont paradoxaux : alors que le mouvement a connu de nombreux succès importants en matière de droits et de changement social, en choisissant l'institutionnalisation plutôt que la politique sexuelle, il a progressivement perdu sa base et son pouvoir politique. Marche suit une approche qu'il décrit comme microsociologique "par le bas " qui se concentre moins sur la reconstruction d'histoires de militance collective que sur les expériences subjectives de ces militant.e.s. Sa source principale est un ensemble de 33 cas tirés d'une base de données de 173 entretiens qualitatifs avec une gamme de différent.e.s militant.e.s LGBT dans les villes de Boston, New York et San Francisco entre 1993 et 1998. La proposition générale est qu'il y a une corrélation entre la politisation subjective de la sexualité, la mobilisation de la base militante et le pouvoir politique relatif du mouvement LGBT. Il conclut que le mouvement LGBT américain a perdu son pouvoir politique parce que la 
source la plus importante de son pouvoir symbolique, la sexualité, a été évacuée du mouvement.

2 Dans les deux premiers chapitres, Marche prépare le terrain avec une chronologie du processus de la désexualisation du mouvement. Au chapitre 1 « Des homosexualités et des mouvements ", il détermine deux points de retournement historique qui ont façonné le mouvement (la fin des décennies 1960 et 1990) et trois orientations fondamentales de ce mouvement social: dans les années 1950, le mouvement homophile a mis l'accent sur la respectabilité et les droits civiques ; dans le milieu des années 1970, le communautarisme gay a mené la privatisation de l'identité sexuelle et la formation des institutions collectives ; mais, c'est la libération gay à la fin des années 1960 qui a insisté sur la politisation de la sexualité. D'après Marche, ce troisième courant a réussi parce que, en apportant la culture sexuelle des bars dans les rues, il contestait fondamentalement sa marginalisation sexuelle. Ce courant a ensuite été rallumé par l'activisme de ACT UP autour du sida à la fin des années 1980 en rapportant une politique radicale du mouvement LGBT. Toutefois, le milieu des années 1990 a donné lieu à un ensemble d'événements politiques qui ont souligné l'institutionnalisation du mouvement à l'échelle nationale. Cette époque fait l'objet de l'analyse plus ciblée du chapitre 2 "Entre éclatement et coalescence ». Maintenant plus optimiste à l'ère des cocktails anti-VIH et des Clintons, le mouvement a choisi des luttes de droits comme les inclusions militaires et le mariage entre personnes de même sexe. Mais, comme le soutient Marche, ces occasions politiques ont créé un autre paradoxe pour le mouvement: pour en profiter, il nécessitait la minimisation de la stigmatisation sexuelle du mouvement ; en minimisant la sexualité, il a perdu sa composante unificatrice et, donc, son pouvoir politique.

3 Dans les chapitres 3, 4 et 5, Marche analyse ces entrevues pour déterminer comment les militant.e.s se sentaient et réagissaient à ces changements dans le mouvement plus institutionnalisé des années 1990. Le chapitre 3, «Épanouissement sexuel et déception politique », décrit leurs sentiments de déception par rapport au mouvement et leur retraite de la militance organisée. Ici, Marche constate que la désexualisation de la politique LGBT a créé un sentiment d'aliénation, développé en tandem avec la marchandisation des identités LGBT à travers le marché gay, le village gay et la dépolitisation de la "gay pride». Repoussés par le manque d'authenticité du mouvement et ses nouvelles normes, les anciens militants se réfugient dans la nouvelle politique de la citoyenneté sexuelle privée. Le chapitre 4, "Sexualité et empowerment » se concentre davantage sur les pratiques plus décentralisées, telles que des projets d'éducation sexuelle dirigés par des pairs, des groupes de soutien social ou même des groupes d'action directe temporaires, qui ont permis aux participants de réinvestir dans la politique sexuelle pendant cette période. Enfin, le chapitre 5, "Agir politiquement aux limites du politique», explore le rôle des modalités « infrapolitiques », des pratiques culturelles qui reposent souvent sur le « camp » et qui n'ont pas d'idéologie ni d'objectif direct. L'auteur fournit, par exemple, un portrait détaillé de ces modalités à travers ses entretiens avec Les Sœurs de la Perpétuelle Indulgence.

4 Bien que ce livre soit excellent dans l'ensemble, il y a certains éléments qui nécessitent une réflexion plus approfondie. Premièrement, l'idée que la sexualité elle-même est la force unificatrice du mouvement LGBT semble initialement une logique issue de l'expérience masculine gay. Sans évoquer la question de la place des personnes 
transgenres dans l'acronyme LGBT, cette thèse n'est pas directement applicable aux lesbiennes non plus, surtout les lesbiennes radicales selon lesquels la lesbienne est une catégorie politique plutôt qu'une identité sexuelle. Deuxièmement, il y a une tension intéressante entre la thèse de l'auteur et les conclusions du chapitre $3:$ tandis que l'auteur a constaté que le mouvement a perdu sa base en raison de sa propre orientation politique, les témoignages suggèrent que la marchandisation des identités LGBT a été aussi très importante. Cette évidence mérite d'être examinée de façon plus approfondie. Troisièmement, la source principale pour ce livre est un ensemble d'entretiens qualitatifs menés avec les militant.e.s LGBT il y a plus de vingt ans. Ce ne sont pas des histoires orales. Même si la façon dont Marche a tissé ce livre autour de ces artefacts des années 1990 est impressionnante, il est nécessaire de relever les défis que pose l'utilisation d'une telle source.

Nonobstant ces réserves, La militance LGBT aux États-Unis offre beaucoup plus qu'implique son titre modeste. Il s'agit d'une contribution approfondie et perspicace à la sociologie gay, à l'histoire des LGBT et à l'étude du militantisme et des mouvements sociaux. À une époque où le mouvement LGBT américain semble avoir perdu tellement de terrain, il pose une question qui préoccupe actuellement de nombreux militant.e.s et chercheur.e.s. Il manifeste puissamment les coûts du choix de l'homonormativité plutôt que la politisation continue de la sexualité. Sa chronologie apporte également une contribution unique et importante à notre compréhension des relations complexes entre les revendications assimilationnistes du mouvement pour les droits et les tentatives des militant.e.s LGBT de réaffirmer une version plus politisée de la sexualité. Son analyse de l'expérience subjective des militant.e.s offre une approche unique et riche pour comprendre l'histoire de ce mouvement. Ses récits détaillés des expériences des militant.e.s offrent un modèle pour changer l'échelle des activismes que nous étudions en considérant l'importance symbolique des petits groupes, des actes provisoires et des performances corporelles. Et bien qu'il ne puisse pas répondre complètement à la question de ce qui est arrivé au mouvement LGBT américain, il établit un cadre afin que d'autres chercheurs puissent poursuivre cette analyse.

\section{AUTEURS}

\section{JULIE PODMORE}

Professeure adjointe au département de géographie, urbanisme et environnement de l'Université Concordia et professeure de géographie au Collège John Abbott. Elle est aussi membre de la Chaire de recherche sur l'homophobie de l'Université du Québec à Montréal.

julie.podmore@concordia.ca 